\title{
Ultrasonic surface rolling process induced elastic-plastic stress wave
}

\author{
Yu Liu ${ }^{1, a^{*}}$ \\ ${ }^{1}$ School of Mechanical Science and Engineering, Jilin University, Changchun 130025, China \\ aliuyuu@jlu.edu.cn
}

Keywords: Ultrasonic Surface Rolling Process; Elastic Stress Wave; Plastic Stress Wave

Abstract. Ultrasonic surface rolling process (USRP) as a novel surface nanocrystallization method generates severe plastic deformation in material surface through combined static extrusion and dynamic impact. Since the dynamic impact acts at ultrasonic frequency, the elastic-plastic deformation is mainly induced by elastic-plastic stress wave. The present work concentrated on the solution of elastic-plastic stress wave by using characteristics method to solve the governing equations of three-dimensional spherical wave, while the impact velocity as well as the material constitutive relation involved in the process of solving was cited from former study.

\section{Introduction}

Ultrasonic surface rolling process, developed in recent years, is proved to be a very effective way for generating nanocrystalline surface layer, improving hardness, as well as reducing surface roughness [1,2]. Its processing tip accomplishes static extrusion and dynamic impact simultaneously, which induces severe plastic deformation (SPD) in material surface. Studies on SPD methods, such as surface mechanical attrition treatment, high energy shot peening, ultrasonic shot peening, supersonic fine particles bombarding, etc., all focused on the microstructure and properties of material, yet little attention was paid on dynamic response [3]. It is known that the frequency of dynamic impact during USRP is ultrasonic. As a result, the elastic-plastic deformation is mainly induced by elastic-plastic stress wave. Thus, this paper investigated the three-dimensional spherical elastic-plastic wave based on previous research results and aimed to provide reference for subsequent study [3,4].

\section{Elastic stress wave}

For ultrasonic surface rolling process, the contact between processing tip and material surface can be regarded as point contact. Correspondingly, the impact action can be regarded as point impact. The stress wave generated by this point impact belongs to three-dimensional spherical wave. The wave front diffuses in wave propagation process, and the wave profile is also changing. Because of spherical symmetry, in polar coordinates $(r, \theta, \varphi)$, only the radial displacement component $u(r, t)$ is non-zero component, and each state parameter is just the function of spherical diameter $r$ and time $t$, shown in Fig. 1. Set $v$ as particle velocity, in the physical coordinate [5]:

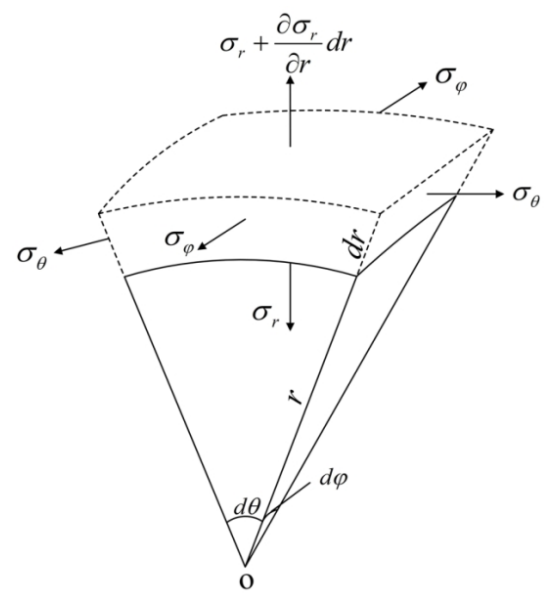

Fig. 1 Infinitesimal body in polar coordinates 


$$
\begin{aligned}
& \varepsilon_{r}(r, t)=\frac{\partial u(r, t)}{\partial r}, \cup(r, t)=\frac{\partial u(r, t)}{\partial t} . \\
& \varepsilon_{\theta}(r, t)=\varepsilon_{\varphi}(r, t)=\frac{u(r, t)}{r} . \\
& \sigma_{r}=\sigma_{r}(r, t), \sigma_{\theta}(r, t)=\sigma_{\varphi}(r, t) .
\end{aligned}
$$

The governing equations of stress wave include continuity equations, equations of motion and material constitutive equations. According to consistency condition, the continuity equations of spherical wave are [5]:

$$
\begin{aligned}
\frac{\partial \varepsilon_{r}}{\partial t} & =\frac{\partial v}{\partial r} . \\
\frac{\partial \varepsilon_{\theta}}{\partial t} & =\frac{v}{r} .
\end{aligned}
$$

Set $\rho_{0}$ as the density of material, equations of motion for spherical wave are:

$$
\frac{\partial \sigma_{r}}{\partial r}+\frac{2\left(\sigma_{r}-\sigma_{\theta}\right)}{r}=\rho_{0} \frac{\partial v}{\partial t} \text {. }
$$

When the stress is below yield limit, stress wave is elastic. Assuming the material to be linear elastic, the constitutive relation follows the generalized Hooke's law. Thus, the constitutive equations are as follows:

$$
\begin{aligned}
& \sigma_{r}+2 \sigma_{\theta}=3 K\left(\varepsilon_{r}+2 \varepsilon_{\theta}\right) . \\
& \sigma_{r}-\sigma_{\theta}=2 G\left(\varepsilon_{r}-\varepsilon_{\theta}\right) .
\end{aligned}
$$

where $K$ is bulk modulus, $K=E / 3(1-2 v) ; E$ is elastic modulus; $v$ is Poisson's ratio; and $G$ is shear modulus, $G=E / 2(1+v)$. Eliminate the strain item from Eq. 4, Eq. 5, Eq. 7 and Eq. 8:

$$
\begin{aligned}
& \frac{1}{3 K} \frac{\partial \sigma_{r}}{\partial t}+\frac{2}{3 K} \frac{\partial \sigma_{\theta}}{\partial t}-\frac{\partial v}{\partial r}-\frac{2 v}{r}=0 . \\
& \frac{1}{2 G} \frac{\partial \sigma_{r}}{\partial t}-\frac{1}{2 G} \frac{\partial \sigma_{\theta}}{\partial t}-\frac{\partial v}{\partial r}+\frac{v}{r}=0 .
\end{aligned}
$$

Eq. 6, Eq. 9 and Eq. 10 are hyperbolic first-order partial differential equations with $\sigma_{r}, \sigma_{\theta}$ and $v$ as unknown function. The characteristics method was adopted to solve the partial differential equations, and the linear combination of these equations should be able to convert to directional derivative only along characteristic line. Three characteristic line equations and corresponding characteristic relations are:

$$
\begin{aligned}
& d r=0 . \\
& \left(\frac{1}{3 K}-\frac{1}{2 G}\right) d \sigma_{r}+\left(\frac{2}{3 K}+\frac{1}{2 G}\right) d \sigma_{\theta}=\frac{3 v}{r} d t . \\
& d r= \pm C_{L} d t . \\
& d \sigma_{r}= \pm \rho_{0} C_{L} d v-2\left[\left(\sigma_{r}-\sigma_{\theta}\right) \mathrm{m}\left(K-\frac{2 G}{3}\right) \frac{v}{C_{L}}\right] \frac{d r}{r} .
\end{aligned}
$$

Here, the elastic wave velocity $C_{L}^{e}$ is one-dimensional strain elastic wave velocity, and the elastic modulus is confining elastic modulus in one-dimensional strain state:

$$
\begin{aligned}
& C_{L}^{e}=\sqrt{\frac{E_{L}}{\rho_{0}}}=\sqrt{\frac{1}{\rho_{0}} \frac{(1-v) E}{(1+v)(1-2 v)}} . \\
& E_{L}=K+\frac{4}{3} G=\frac{(1-v) E}{(1+v)(1-2 v)} .
\end{aligned}
$$

The first group of characteristic lines represents the particle trajectory. The compatible condition along these characteristic lines is the differential form of any particle that meets the constitutive relation. The other two groups of characteristic lines represent the propagation path of forward and negative 
wave matrix surface. Relevant compatibility relation specifies the constraint relationship among $\sigma_{r}, \sigma_{\theta}, \mathrm{v}$. In general, the impacted sample is static in initial state during USRP. That is $\sigma_{0}=\varepsilon_{0}=\mathrm{v}_{0}$. At $t=0$, the first point of contact $(r=0)$ between the sample surface and processing tip suffers a given conditional impact. It has been known that the relationship of particle velocity changing with time is $v(t)=0.96 \sin (125664 t-\pi) t \geq 0$ at the contact point [4]. Thus, the initial condition is:

$$
v(r, 0)=\varepsilon(r, 0)=0,0<r \leq \infty \text {. }
$$

The boundary condition is:

$$
v(0, t)=v_{0}(\tau)=0.96 \sin (125664 t-\pi), t \geq 0 .
$$

Based on Eq. 18, the boundary condition belongs to weak discontinuous boundary condition. Hence, the elastic spherical wave belongs to weak discontinuous wave, namely continuous wave. The distribution of stress, strain and particle velocity in semi infinite body at any moment, as well as the variation of them with time at any position can be determined by characteristic line graphic method, shown in Fig. 2.

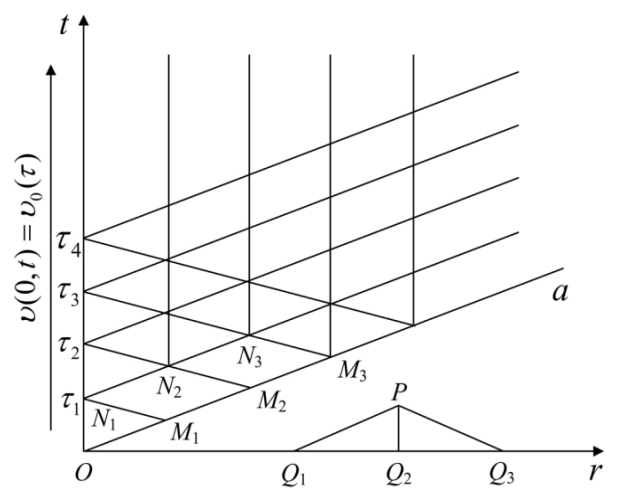

Fig. 2 The characteristic line graphic method to solve the problem of elastic spherical wave

In $(r, t)$ plane, there are three intersecting characteristic lines at any point. $O a$ is the characteristic line through the original point, which divides the entire physical plane into two areas. The essence of solving elastic spherical wave comes down to solve the problem of Cauchy initial value in roa region and characteristics boundary value in aot region. For roa region, the particle velocity and strain along or axis can be known from initial conditions. Three characteristic lines through any single point $P$ in $r o a$ region all intersect with $o r$ axis, and the intersection point is $Q_{1}, Q_{2}, Q_{3}$, respectively. If the distance between point $P$ and $r$ axis is sufficiently small, the distance between point $P$ and $Q_{1}, Q_{2}, Q_{3}$, is also small enough. Then, difference can be used to substitute for differential. According to the compatibility relation, it has:

$$
\begin{aligned}
& \sigma_{r}(P)-\sigma_{r}\left(Q_{1}\right)=\rho_{0} C_{L}^{e}\left[v(P)-v\left(Q_{1}\right)\right]-2\left[\left(\sigma_{r}-\sigma_{\theta}\right)-\left(K-\frac{2 G}{3}\right) \frac{v}{C_{L}^{e}}\right] \cdot \frac{r(P)-r\left(Q_{1}\right)}{r\left(Q_{1}\right)} . \\
& \sigma_{r}(P)-\sigma_{r}\left(Q_{3}\right)=-\rho_{0} C_{L}^{e}\left[v(P)-v\left(Q_{3}\right)\right]-2\left[\left(\sigma_{r}-\sigma_{\theta}\right)+\left(K-\frac{2 G}{3}\right) \frac{v}{C_{L}^{e}}\right] \cdot \frac{r(P)-r\left(Q_{3}\right)}{r\left(Q_{3}\right)} . \\
& \left(\frac{1}{3 K}-\frac{1}{2 G}\right)\left[\sigma_{r}(P)-\sigma_{r}\left(Q_{2}\right)\right]+\left(\frac{2}{3 K}+\frac{1}{2 G}\right)\left[\sigma_{\theta}(P)-\sigma_{\theta}\left(Q_{2}\right)\right]=\frac{3 v\left(Q_{2}\right)}{r\left(Q_{2}\right)}\left[t(P)-t\left(Q_{2}\right)\right] .
\end{aligned}
$$

Ascertain the location of point $P$ through Eq. 11 and Eq. 13, and then based on the initial condition $v(r, 0)=\varepsilon(r, 0)=0, \sigma_{r}\left(Q_{1}\right)=\sigma_{r}\left(Q_{2}\right)=\sigma_{r}\left(Q_{3}\right)=\sigma_{\theta}\left(Q_{1}\right)=\sigma_{\theta}\left(Q_{2}\right)=\sigma_{\theta}\left(Q_{3}\right)=v\left(Q_{1}\right)=v\left(Q_{2}\right)=v\left(Q_{3}\right)=0$ can be obtained. Integrate Eq. 19, Eq. 20 and Eq. 21, $v(P)=\varepsilon(P)=0$ can be finally obtained. Since $P$ is a random point in roa region, $v=\varepsilon=0$ will be tenable in the entire roa region, which means that the roa region is a zero constant value region. For aot region, the variation of particle velocity at the contact point between sample surface and processing tip along with time is provided by boundary condition. Pick a point $N_{1}$ on ot axis to keep the distance between $N_{1}$ and $M_{1}$ small enough. Using the same way in solving the roa region, calculate the difference along the characteristic line $M_{1} N_{1}$ and $O N_{1}$ through Eq. 12 and Eq. 14: 


$$
\begin{aligned}
& \sigma_{r}\left(N_{1}\right)-\sigma_{r}\left(M_{1}\right)=-\rho_{0} C_{L}^{e}\left[v\left(N_{1}\right)-v\left(M_{1}\right)\right]-2\left[\left(\sigma_{r}-\sigma_{\theta}\right)+\left(K-\frac{2 G}{3}\right) \frac{v}{C_{L}^{e}}\right] \cdot \frac{r\left(N_{1}\right)-r\left(M_{1}\right)}{r\left(M_{1}\right)} . \\
& \left(\frac{1}{3 K}-\frac{1}{2 G}\right)\left[\sigma_{r}\left(N_{1}\right)-\sigma_{r}(O)\right]+\left(\frac{2}{3 K}+\frac{1}{2 G}\right)\left[\sigma_{\theta}\left(N_{1}\right)-\sigma_{\theta}(O)\right]=\frac{3 v(O)}{r(O)}\left[t\left(N_{1}\right)-t(O)\right] .
\end{aligned}
$$

The solution along characteristic line $o a$ can be obtained from solving the roa region. Meanwhile, the particle velocity $v\left(N_{1}\right)$ at $N_{1}$ can be worked out through Eq. 18. Therefore, $\sigma_{r}\left(N_{1}\right)$ and $\sigma_{\theta}\left(N_{1}\right)$ can be solved by Eq. 22 and Eq. 23. After calculating the point $N_{1}$, point $N_{2}$ can be further calculated with the known points $M_{1}, M_{2}$. On the analogy of this, particles in the whole aot region all can be calculated. It is worth noting that the velocity calculated through Eq. 18 contains the whole movement of processing tip. The downward movement belongs to loading, and the solution of elastic stress wave can be carried out in accordance with the above method, while the upward movement belongs to unloading, and although the solution of elastic unloading wave can also be carried out in accordance with the above method owing to the unchanged material constitutive relation, the effect on plastic wave will change.

\section{Plastic stress wave}

Assume that plastic deformation makes no contribution to volume deformation. When the stress exceeds yield limit, plastic deformation occurs in addition to elastic deformation, which will generate elastic-plastic stress wave. Thus, the plastic part should be considered in material constitutive relation. For as-received $40 \mathrm{Cr}$ steel, due to spherical symmetry Mises yield criterion in 3-d polar coordinate can be turned into the form below:

$$
\sigma_{r}-\sigma_{\theta}= \pm Y= \pm\left\{443.3+872.1 \varepsilon^{p}+234.7\left[1-\exp \left(-29.23 \varepsilon^{p}\right)\right]\right\} \text {. }
$$

where $Y$ is yield function. The rate-independent constitutive equations of elastic-plastic spherical wave governing equations in the form of capacitance change law and distortion law are:

$$
\begin{aligned}
& \sigma_{r}+2 \sigma_{\theta}=3 K\left(\varepsilon_{r}+2 \varepsilon_{\theta}\right) . \\
& \sigma_{r}-\sigma_{\theta}=2 G\left(\varepsilon_{r}-\varepsilon_{\theta}\right),(\text { elastic }) . \\
& \sigma_{r}-\sigma_{\theta}= \pm\left\{443.3+872.1 \varepsilon^{p}+234.7\left[1-\exp \left(-29.23 \varepsilon^{p}\right)\right]\right\},(\text { plastic }) .
\end{aligned}
$$

Except for Eq. 6 and Eq. 9, the governing equations of plastic spherical wave also include:

$$
\frac{1}{2 G_{p}} \frac{\partial \sigma_{r}}{\partial t}-\frac{1}{2 G_{p}} \frac{\partial \sigma_{\theta}}{\partial t}-\frac{\partial v}{\partial r}+\frac{v}{r}=0
$$

where $G_{p}$ is plastic shear modulus, half of the slope of material plastic curve.

$$
G_{p}=\frac{G Y^{\prime}}{3 G+Y^{\prime}}=\frac{G\left(872.1+6860.3 \exp \left(-29.23 \varepsilon_{p}\right)\right)}{3 G+872.1+6860.3 \exp \left(-29.23 \varepsilon_{p}\right)} .
$$

where $Y^{\prime}$ is the derivative of stress vs. plastic strain.

Hence, Eq. 6, Eq. 9 and Eq. 28 constitute the hyperbolic first-order partial differential equations with $\sigma_{r}, \sigma_{\theta}$ and $v$ as unknown function. The characteristic line method is still used to solve the plastic spherical wave problems. Its three characteristic lines and corresponding compatible relationships are:

$$
\begin{aligned}
& d r=0 . \\
& \left(\frac{1}{3 K}-\frac{1}{2 G_{p}}\right) d \sigma_{r}+\left(\frac{2}{3 K}+\frac{1}{2 G_{p}}\right) d \sigma_{\theta}=\frac{3 v}{r} d t . \\
& d r= \pm C_{L}^{P} d t . \\
& d \sigma_{r}= \pm \rho_{0} C_{L}^{P} d v-2\left[\left(\sigma_{r}-\sigma_{\theta}\right) \mathrm{m}\left(K-\frac{2 G_{P}}{3}\right) \frac{v}{C_{L}^{P}}\right] \frac{d r}{r} .
\end{aligned}
$$


Besides, the velocity of plastic spherical wave is:

$$
C_{L}^{P}=\sqrt{\left(K+\frac{4}{3} G_{P}\right) / \rho_{0}} .
$$

It can be seen from Eq. 34 that the wave velocity $C_{L}^{P}$ is determined by the density of material $\rho_{0}$ and the slope of plastic section of $\sigma-\varepsilon$ curve $d \sigma / d \varepsilon$, which means that strain hardening characteristics have great influence on wave propagation. For material of which the tangent modulus decreases with the increase of strain $\left(d^{2} \sigma / d \varepsilon^{2}<0\right)$, the plastic wave velocity decreases with the increase of strain. When $40 \mathrm{Cr}$ steel is processed by USRP, the propagation speed of high amplitude perturbation is less than that of the ahead low amplitude perturbation. Therefore, in the process of stress wave propagation, the wave profile gradually tends to be smooth. Combined with the known weak discontinuous boundary conditions, the plastic spherical wave generated by the processing tip impacting $40 \mathrm{Cr}$ surface is weak discontinuity plastic wave, namely the continuous wave.

Also, it can be found from Eq. 34 that $C_{L}^{P}$ is the function of strain $\varepsilon_{r}^{P}$. On physical plane, two groups of characteristic line characterizing plastic spherical wave propagation are no longer straight (except ideal plastic material and linear hardening material). The solving method of plastic spherical wave loading problem can be made from the extension of elastic spherical wave discussion.

\section{Summary}

The elastic-plastic stress wave generated during USRP was studied. The governing equations of stress wave were solved through characteristics method. By using previous research results, the elastic stress wave during downward impact and upward rebound can be calculated. Also, the solving method of plastic stress wave was presented, and it was found that the processing tip impacting 40Cr surface produces weak discontinuity plastic wave.

\section{Acknowledgement}

This research was financially supported by the National Science Foundation through grant no. 51405182 and the Youth Scientific Research Fund of Jilin Province through grant no. 20130522184JH.

\section{References}

[1] T. Wang, D.P. Wang, G. Liu, B.M. Gong, N.X. Song, Investigations on the nano-crystallization of 40Cr using ultrasonic surface rolling processing, Appl. Surf. Sci., 255 (2008) 1824-1829.

[2] X.H. Zhao, Y.J. Fan, H.Y. Wang, Y. Liu, Revealing the surface nano-enhancing mechanism of $\alpha$-titanium alloy by microstructure evolution, Mater. Lett., 160 (2015) 51-54.

[3] Taku Sakai, Andrey Belyakov, Rustam Kaibyshev, Hiromi Miura, John J. Jonas, Dynamic and post-dynamic recrystallization under hot, cold and severe plastic deformation conditions, Prog. Mater Sci., 60 (2014) 130-207.

[4] X.H. Zhao, Y. Liu, Investigation on Dynamics of Ultrasonic Surface Rolling Process, Advanced Materials Research, 834-836 (2014) 640-643.

[5] L.L. Wang, Foundation of Stress Waves, second ed., Beijing, 2005. 